\title{
Evidence for Positive Effects of Date Extract That Attenuates Thermal Hyperalgesia in a Diabetic Rat Model of Neuropathic Pain
}

\author{
Mohammad Shabani $^{1}$, Nasser Zangiabadi ${ }^{1,2^{*}}$, Majid Asadi-Shekaari ${ }^{1}$ \\ ${ }^{1}$ Neuroscience Research Center, Kerman University of Medical Sciences, Kerman, Iran; ${ }^{2}$ Afzal Research Center, Kerman, Iran. \\ Email: "nzangiabadi1@gmail.com
}

Received January $18^{\text {th }}$, 2013; revised February $20^{\text {th }}, 2013$; accepted February $28^{\text {th }}, 2013$

\begin{abstract}
Aim: Diabetic neuropathic pain is one of the pains which hardly respond to pharmaceutical treat. Today, various chemical and herbal compounds have been used to reduce pain. The aim of this study is to compare the effect of date extract and melatonin in preventing pain in diabetic rats. Method: To study hyperalgesia response and to compare the effect of date extract and melatonin in preventing pain, hot plate and tail flick tests were used. After prescribing single dose of streptozotocin to rats and approving their diabetes, treatment rats received date extract $(4 \mathrm{ml} / \mathrm{kg} /$ day $)$ or melatonin $[10 \mathrm{mg} / \mathrm{kg} / \mathrm{day}$, intraperitoneally (i.p.)] for a period of 6 weeks. At the end of the sixth week, control and treated rats were examined by thermal pain response and explorative activity tests. Results: According to hot plate results, response time to thermal pain in treated group showed a significant decrease in comparison with the control group (P< 0.01). Prescription of date extract increased response time to thermal pain in comparison with treated group $(\mathrm{P}<0.01)$, so that response time approximated to control group. Although melatonin approximated to the response time to control group, the significant difference was not observed among melatonin receivers and other groups. In the assessment of diabetic neuropathy on the explorative activity of rats in an open field behavioral test, total distance moved and rearing frequency were significantly decreased, while administration of date extract did also improve motor deficits induced by STZ. Conclusions: Findings of this study showed that date extract decreased thermal hyperalgesia and can prevent pain resulted from diabetic neuropathy.
\end{abstract}

Keywords: Date Extract; Diabetic; Melatonin; Neuropathic Pain; Thermal Hyperalgesia

\section{Introduction}

Neuropathic pain is one of the most common and painful symptoms of diabetic neuropathic pain which is present in both I and II types of diabetes [1,2]. Neuropathy resulted from diabetes occurs mostly with hyperalgesia and allodynia [3]. Although several studies have reported destruction of nerves or changes in neurotransmitters release as the cause of changes in perceiving pain in diabetic patients, its exact mechanism is still unknown $[4,5]$. Side effects of chemical drugs that used in decreasing pain cause increase using of herbal drugs; and this type of treatment has been taken into consideration. Some studies have shown that diabetes mellitus occurs mainly with chronic neuropathic pain, and oxidative stress plays an important role in emergence of most Neurologic and behavioral changes in diabetic patients [6,7]. Therefore, studying the effect of antioxidants on releasing diabetic neuropathic pain is of great importance.

${ }^{*}$ Corresponding author.
Concerning confirmed antioxidant effect, date extract [8] and melatonin [6,7] can be regarded as the choices in reducing neuron damages and neuropathic pain resulted from diabetes. Melatonin reduces pain through connecting to MT1 and MT2 receptors [6,9] and then reducing the production of cAMP [10]. Also, melatonin has been shown to activate opioid receptors indirectly and thus reduces the pain [11]. Melatonin can reduce pain in other ways including inhibiting cyclooxygenase and preventing the production of free radicals, and can be used as a drug for reducing neuropathic pain [12].

Today, the powerful antioxidant effect of such food supplementary has been recognized. One of these foods supplementary is date which is of the Palmaceae family named "phoenix Dactylifera”. It has antioxidant [13] and antibacterial effects; its effect on immune system has been recognized [14]. Due to sugar contents, vitamins, fatty acids and proteins, date is considered very important in human nutrition [15]. Regarded to its anti-pain effects in traditional medicine, it is used for backaches, 
rheumatic pains, cough and chest pain [16]. Regarding the anti-inflammatory, antioxidant and anti-pain effects of date extract and melatonin and concerning their roles in forming free radicals and cytokines, this study was carried out to determine the effect of date extract and melatonin in preventing neuropathic pains and also the probable anti-pain effect of these two materials after creating diabetes model in male rats.

\section{Materials and Methods}

This study was carried out on male rats of Sprague Dawley race weighting 200 - 250 gram. During treatment period, the animals were housed under optimal laboratory conditions, maintained on a natural light and dark cycle and had a free access to food and water ad libitum. Animals were acclimatized to laboratory conditions before the tests. All experiments were carried out between 09:00 and13:00 h.

These rats were divided into four groups: First group consists of control animals, second, third and fourth groups consisted of diabetic animals treated with melatonin (10 mg/kg/day for 6 weeks, i.p.), date extract and vehicle ( $4 \mathrm{ml} / \mathrm{kg} /$ day, orally), at least 8 rats in every group.

\subsection{Diabetes Induction Method}

Diabetes induction was done by $45 \mathrm{mg}$ intra peritoneal injection of STZ per kg, solved in citrate buffer $0.05 \mathrm{M}$. Blood sugar measurement was done for diagnosing diabetes induction at the end of the first week after injecting STZ using tail's vein blood (by ACCU-CHEK-ACTIVE kit made in German Roche Company). The rats with blood sugar over $200 \mathrm{mg} / \mathrm{dl}$ were considered diabetic [17]. Slowing of sensory nerve conduction velocity and motor nerve conduction velocity, hyperalgesia and allodynia have shown to develop within the first month of the onset of hyperglycemia in STZ rats [18,19]. Thus, in this study, to prevent neuropathic pain, melatonin $(10 \mathrm{mg} / \mathrm{kg}$, daily as intra peritoneal injection) and date extract $(4 \mathrm{ml} / \mathrm{kg}$, daily as gavage) were used for a period of 6 weeks, after rats were confirmed diabetic. Such as date extract group, vehicle group also received distilled water from the end of first week for a period of six weeks.

\subsection{Pain Evaluation Method in Two Models of Tail Flick and Hot Plate}

\subsubsection{Anti-Pain Response Measurement in Test Tail Flick}

Tail Flick Test is one of the standard tests for measuring Analgesia amount. In this test, thermal light was shone to the end of tail of rats with intensity of 5 using tail flick machine (made by Spanish Lsi LETICA, model LE7406) and tail flick latency was measured from the light emis- sion to take the tail (on second). To prevent tissue damage, maximum light mission to the tail was considered 10 seconds. For every rat, tail flick latency (TFL) was measured for three times and the average was reported as TFL. Between every test, rats were left free for half an hour [20].

\subsubsection{Anti-Pain Response Measurement in Test Hot Plate}

Another tool used to measure sensitivity to pain was Hot Plate Machine (made by Spanish Lsi LETICA, model LE7406) which consisted of a $19 \mathrm{~cm}$-diameter plate and a plexi glass wall with height of $30 \mathrm{~cm}$. it heats up through electric resistance and is equipped with a timer and a thermostat. The plate thermal degree was adjusted on $52^{\circ} \mathrm{C} \pm 2^{\circ} \mathrm{C}$. Thermal pain response time was calculated from the beginning of test to the time when the animal started licking its front legs or jumping. Maximum time against thermal pain was considered 60 seconds [21,22].

\subsubsection{Open Field Test}

The horizontal and vertical activities of the male rats were recorded for a period of 5 min and then analyzed using Ethovision software [version 7.1], a video tracking system for automation of behavioral experiments [Noldus Information Technology, the Netherlands]. The apparatus consisted of a square arena $[56 \times 56 \times 20[\mathrm{H}] \mathrm{cm}]$ made of black wood and its floor was divided by lines into 16 squares that allowed the definition of central and peripheral parts. At the beginning of the session, each rat was placed in the centre of the arena and its activity was recorded for $5 \mathrm{~min}$ and the following behavioral parameters were then scored: total distance moved [TDM, cm]; total duration of mobility [s] and immobility [s]; and frequency of rearing [as a measure of vertical activity]. At the end of each session, rats were removed from the open field and the experimental chamber was thoroughly cleaned with a damp cloth and dried [22-24].

\subsubsection{Drugs Preparation}

To prepare date extract, fresh Phoenix Dactylifera was prepared from Bam Town Orchards, Kerman Province (South East of Iran), the kernel was removed and 100gr of date was immersed in $1000 \mathrm{~mL} /$ distilled water for 48 hours. After that, it was mixed completely in a mixer and then the mixture was centrifuged in $4^{\circ} \mathrm{C}$ for 20 minutes and with $4000 \mathrm{rpm}$. After sedimentation, the impurities existing in upper parts were used for gavage [25].

\subsection{Statistical Analysis Method}

Data was presented as mean and standard error of mean (SEM). To study the statistical difference, analysis test 
and one-way ANOVA were used. In the case of significant, to study the difference among groups, Tukey post hoc test was used. $\mathrm{P}<0.05$ was considered as significant level.

\section{Results}

\subsection{Study the Effect of Date Extract in Preventing Diabetic Neuropathic Pain in Hot Plate Test}

In this study, reaction time to thermal pain decreased significantly in vehicle taking diabetic group in comparison with control group (6.97 \pm 0.8 vs $3.7 \pm 0.19$, respectively, $\mathrm{P}<0.01)$. Making use of date extract for a period of 6 weeks in gavage, increased reaction time to pain in Hot Plate test in diabetic rats with STZ in comparison with vehicle receiving diabetic group, ( $6.9 \pm 1.2$ vs $3.7 \pm 0.19$, respectively, $\mathrm{P}<0.05$ ), while no significant change was observed in reaction time to thermal pain in date extract group in comparison with control group (Figure 1).

\subsection{Study the Effect of Melatonin in Preventing Diabetic Neuropathic Pain in Hot Plate Test}

Unlike date extract, melatonin intra injection peritoneal for a period of 6 weeks didn't result in a significant change in comparison with vehicle group regarding reaction time to thermal pain, although reaction time to thermal pain in melatonin group was close to that of control group and a significant decrease was found in vehicle group compared to control group (Figure 2).

In Tail Flick Test, in reviewing tail flick time, no significant difference was observed among vehicle receiving diabetic group, melatonin and date extract. In both

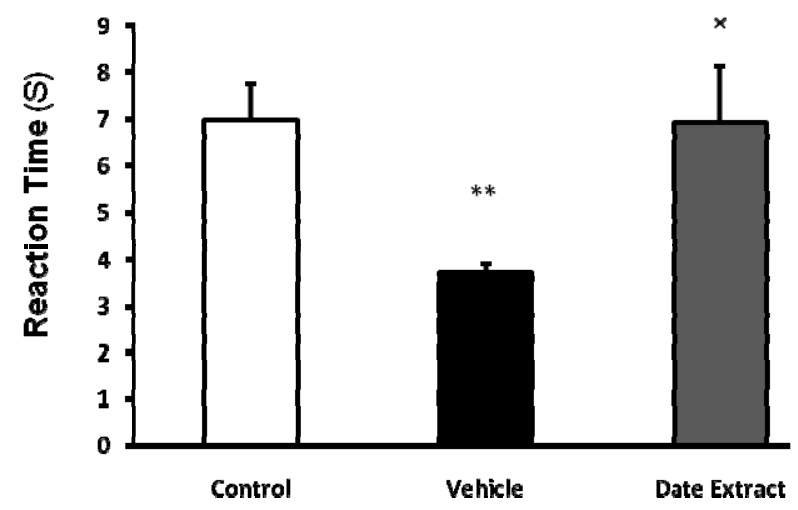

Figure 1. Effect of Date Extract $(4 \mathrm{ml} / \mathrm{kg} / \mathrm{day}$, p.o. for 6 weeks) on the pain threshold values in streptozotocin- injected diabetic rats on the hot plate $\left(52^{\circ} \mathrm{C} \pm 2^{\circ} \mathrm{C}\right)$. Values are expressed as mean \pm S.E.M. ( $n=8$ rats in each group). ${ }^{* *} \mathbf{P}<$ 0.01 as compared to control group. ${ }^{\times} \mathbf{P}<0.05$ as compared to Vehicle-treated diabetic group.

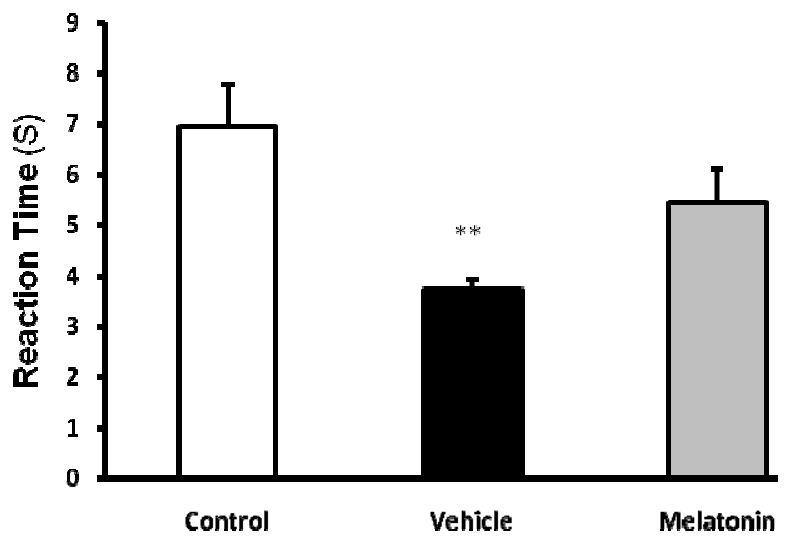

Figure 2. Effect of Melatonin (10 mg/kg/day, ip, for 6 weeks) on the pain threshold values in streptozotocin-injected diabetic rats subjected to hot plate. Values are expressed as mean \pm S.E.M. ( $\mathbf{n}=8$ rats in each group). ${ }^{* *} \mathbf{P}<0.01$ as compared to control group. There are no significance effects between Vehicle and melatonin-treated diabetic groups.

control and diabetic groups, all rats responded to thermal pain in less than 10 seconds and flicked the end of their tails from the painful environment. Making use of date extract and melatonin didn't result in a significant change in reaction time to thermal pain (Figures 3 and 4).

\subsection{Effect of Date Extract and Melatonin on Explorative and Anxiety Related Behaviors in Diabetic Rats}

The open-field test was performed to examine locomotor activity and anxiety-related behavior [26]. Our results showed that STZ did alter some of the parameters evaluated in this test and date extract did have a moderating effect on changes induced by STZ. The rearing frequency $(\mathrm{P}<0.001)$ (Figure 5(a)) and total distance moved (Figure 5(b)) were decreased in vehicle receiving diabetic group compared to control $(\mathrm{P}<0.05)$ and date extract group $(\mathrm{P}<0.05)$. There was no significant difference between control and other treated rats $(\mathrm{P}>0.05)$. There was no significant difference in mobility, immobility and the time spent in center and periphery, indicating no difference in anxiety related behavior (data not show).

\section{Discussion}

In present study, long term consumption of date extract after induction and diabetes proof, showed that date extract can reduce hyperalgesia in response to thermal pain in diabetic animals in a time period similar to control conditions. Unlike date extract, melatonin didn't result in a significant change in comparison with vehicle group regarding reaction time to thermal pain, and it seems that protective effect of date extract is stronger than that of melatonin regarding preventing diabetic neuropathic pain. 


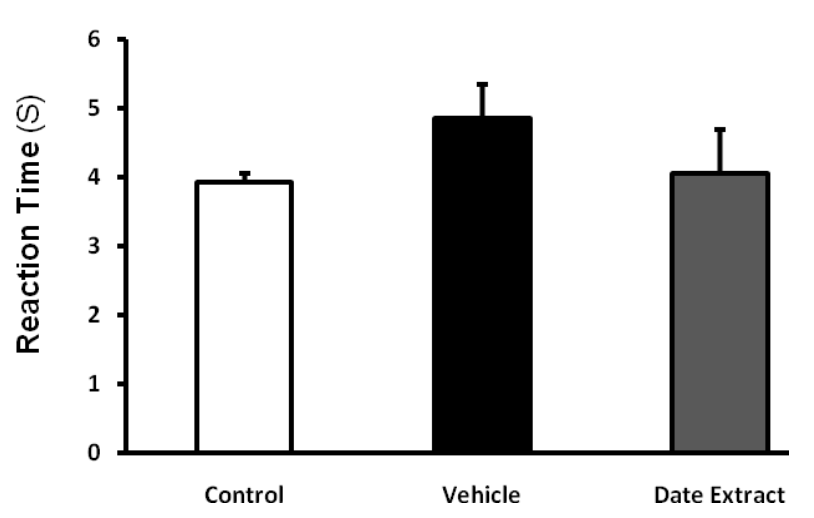

Figure 3. Effect of Date Extract $(4 \mathrm{ml} / \mathrm{kg} / \mathrm{day}$, ip, for 6 weeks) on the pain threshold values in streptozotocin-injected diabetic mice subjected to tail flick. Values are expressed as mean \pm S.E.M. ( $n=8$ rats in each group). There are no significance effects between control group and streptozotocin (STZ)-treated diabetic groups.

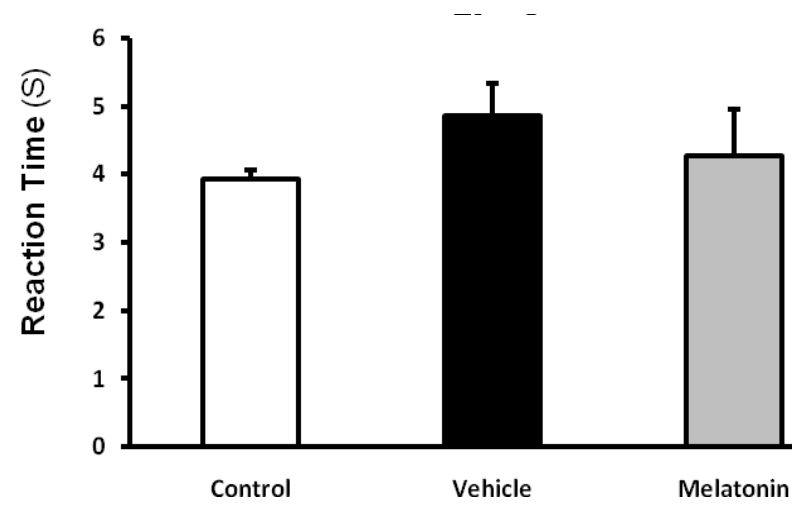

Figure 4. Effect of Melatonin (10 mg/kg/day, ip, for 6 weeks) on the pain threshold values in streptozotocin-injected diabetic rats subjected to tail flick. Values are expressed as mean \pm S.E.M. ( $n=8$ rats in each group). There is no significance effect between any groups.

Environmental neurons damage resulted from oxidative stress in more than $60 \%$ of people with diabetes is recognizable [27]. Environmental neuropathy in the first steps co-occurs with increased activity of nervous fibers and interfered with natural sensitivity of nervous system to painful stimulus and created diabetic hyperalgesia [28]. In some studies, several therapeutic effects of date including strengthening immune-system, anti-bacterial properties [14], anti-oxidant and anti-cancer features have been reported [13]. One of the main clinical complains of people with diabetes mellitus is hyperalgesia resulted from environmental neuropathy which affects the life quality of these people [28]. Therefore, balancing the pain of these patients is of great importance. Thus, study the therapeutic guidelines which can, by reducing oxidative stress conditions and anti-oxidant effects, result in decreased trend of neurons damage and can inhibit

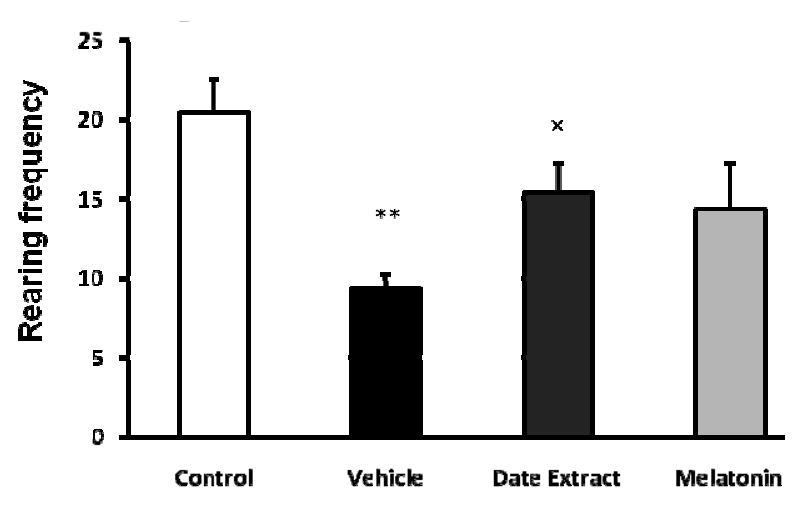

(a)

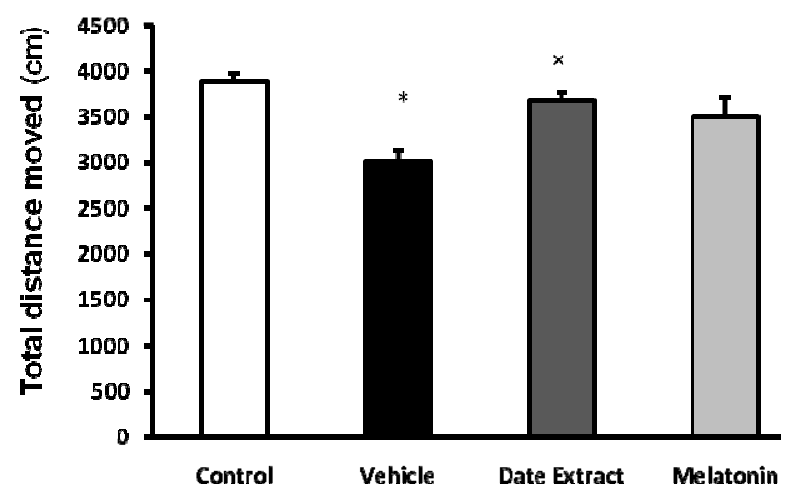

(b)

Figure 5. Effect of Date fruit extract and melatonin on explorative behavior of rats in open field test. (a) Rearing frequency: ${ }^{* *} \mathbf{P}<0.01$ compared with the control group, ${ }^{\dagger} \mathbf{P}<$ 0.05, compared with the Date Extract group; (b) TDM: ${ }^{\times} \mathbf{P}<$ 0.05 compared with the control and Date Extract group. Data are the Mean \pm S.E.M., ( $\mathrm{n}=8$ rats in each group).

hyperalgesia will be very crucial. Melatonin [29,30] and date [8] are among those materials whose anti-oxidant effects and presence of anti-oxidant materials have been proved.

In some studies, anti-pain effect of date has also been introduced and it has been shown that it can reduce backache and rheumatic pains [16]. Also, date has been shown to decrease LDL cholesterol and triglyceride [3133].

Locomotor activity was affected by STZ in the open field test, a decreased level of locomotion was observed in diabetic treated rats, and date extract did modify these alterations to some extent. This decreased locomotion might be due to the fact that STZ treated rats have pain and are weaker and their movement is impaired by this neurotoxic agent.

No exact mechanism has been recognized for antioxidant and oxidative stress reduction effect of date extract. However, these effects are related to date compounds. Compounds like polyphenol, anthocyanin, fla- 
vonoids phenolic acid as well as copper, selenium, zinc and magnesium and vitamin $\mathrm{C}$ can be probably responsible for these therapeutic and protective effects of date [8, $34,35]$. On the other hand, some studies recognized anticancer and anti-oxidant effects of date due to its inhibiting effect on lipid pre-oxidation and protein oxidation. Some studies have revealed that date, in vitro, can eliminate radicals of hydroxyl and super oxide $[8,13]$.

In Tail Flick test, no significant difference was observed in all groups regarding response time to pain; however, in Hot plate test, melatonin increased latency time in response to thermal pain and reduced hyperalgesia so that reaction time to pain in this group approximated to control group, although it had no significant difference with vehicle group. Therefore, it seems that melatonin after diabetes induction for a period of six weeks could, like date extract, prevent diabetic hyperalgesia and increased response time to pain in a time like control group.

There are several studies about useful effects of antioxidants on performance and structure of nervous tissues. In his study, J. Podratz, et al. (2004) showed that presence of anti-oxidants is necessary for melatonin axon of neurons of posterior root [36]. In another study, it was observed that removing anti-oxidants of androgen like vitamin $\mathrm{E}$ in diabetic rats resulted in reduced performance of environmental neurons [37].

Diabetic neuropathy in initial steps increased sensitivity of environmental sensory neurons and central neurons to pain stimuli [38]. In some studies, anti-pain effects from anti-oxidants in diabetic patients have been reported. Prescribing anti-oxidant U83836E is known to decrease diabetic hyperalgesia [39]. It has also been shown that vitamin $\mathrm{E}$ can, as an anti-oxidant, decrease neuropathic pains in diabetic rats [40].

Some studies reported the significant effects of melatonin against oxidative stress in most tissues of diabetic animal [6,7,9,41-43].Consuming melatonin for a long time can prevent neuron degeneration and sensitivity of nervous system in diabetic rats through reduction of ROS surface and free radicals $[6,9]$. Activating opioed recaptors $[44,45]$, inhibiting cyclo-oxygenize and reducing the production of free radicals $[6,9]$, melatonin can play a protective role against neuron damages resulted from devastating trends of diabetes.

To sum up, it seems that using date and melatonin in the first steps of diabetes induction (before neuron destruction and creating of diabetic neuropathy) can reduce neuron destruction resulted from diabetes and approximate the response threshold to pain which is reduced in diabetic neuropathy to normal situation. In this study, it was shown that protective effect of date extract is stronger than that of melatonin regarding preventing diabetic neuropathic pain.

\section{Acknowledgements}

The present manuscript is the product of a research project that was approved by the Neuroscience Research Center in Kerman University of Medical Science; the authors appreciate to the manager of the mentioned center for their financial supports.

\section{REFERENCES}

[1] C. Clark and D. Lee, "Prevention and Treatment of the Complications of Diabetes Mellitus,” New England Journal of Medicine, Vol. 332, No. 18, 1995, p. 1210. doi:10.1056/NEJM199505043321807

[2] S. Sharma, et al., "Curcumin Attenuates Thermal Hyperalgesia in a Diabetic Mouse Model of Neuropathic Pain,” European Journal of Pharmacology, Vol. 536, No. 3, 2006, pp. 256-261. doi:10.1016/j.ejphar.2006.03.006

[3] M. Brown and A. Asbury, "Diabetic Neuropathy,” Annals of Neurology, Vol. 15, No. 1, 1984, pp. 2-12. doi:10.1002/ana.410150103

[4] J. Flier, et al., "Sorbitol, Phosphoinositides, and SodiumPotassium-ATPase in the Pathogenesis of Diabetic Complications,” New England Journal of Medicine, Vol. 316, No. 10, 1987, pp. 599-606. doi:10.1056/NEJM198703053161007

[5] J. Lynch III, M. Jarvis and E. Kowaluk, "An Adenosine Kinase Inhibitor Attenuates Tactile Allodynia in a Rat Model of Diabetic Neuropathic Pain,” European Journal of Pharmacology, Vol. 364, No. 2-3, 1999, pp. 141-146. doi:10.1016/S0014-2999(98)00840-1

[6] N. Aksoy, et al., "Effects of Melatonin on OxidativeAntioxidative Status of Tissues in Streptozotocin Induced Diabetic Rats," Cell Biochemistry and Function, Vol. 21, No. 2, 2003, pp. 121-125. doi:10.1002/cbf.1006

[7] H. Vural, et al., "Melatonin Inhibits Lipid Peroxidation and Stimulates the Antioxidant Status of Diabetic Rats," Journal of Pineal Research, Vol. 31, No. 3, 2001, pp. 193-198. doi:10.1034/j.1600-079X.2001.310301.X

[8] E. Saafi, et al., "Protective Effect of Date Palm Fruit Extract (Phoenix dactylifera L.) on Dimethoate InducedOxidative Stress in Rat Liver," Experimental and Toxicologic Pathology, Vol. 63, No. 5, 2011, pp. 433-441.

[9] P. Montilla, et al., "Oxidative Stress in Diabetic Rats Induced by Streptozotocin: Protective Effects of Melatonin,” Journal of Pineal Research, Vol. 25, No. 2, 1998, pp. 94100. doi:10.1111/j.1600-079X.1998.tb00545.X

[10] E. Peschke, et al., "Receptor (MT1) Mediated Influence of Melatonin on cAMP Concentration and Insulin Secretion of Rat Insulinoma Cells INS 1,” Journal of Pineal Research, Vol. 33, No. 2, 2002, pp. 63-71. doi:10.1034/j.1600-079X.2002.02919.x

[11] R. Arreola-Espino, et al., "Melatonin Reduces FormalinInduced Nociception and Tactile Allodynia in Diabetic Rats,” European Journal of Pharmacology, Vol. 577, No. 
1-3, 2007, pp. 203-210. doi:10.1016/j.ejphar.2007.09.006

[12] D. Tan, et al., “One Molecule, Many Derivatives: A Never Ending Interaction of Melatonin with Reactive Oxygen and Nitrogen Species?” Journal of Pineal Research, Vol. 42, No. 1, 2007, pp. 28-42. doi:10.1111/j.1600-079X.2006.00407.x

[13] P. Vayalil, “Antioxidant and Antimutagenic Properties of Aqueous Extract of Date Fruit (Phoenix dactylifera L. Arecaceae)," Journal of Agricultural and Food Chemistry, Vol. 50, No. 3, 2002, pp. 610-617. doi:10.1021/jf010716t

[14] A. Sallal and A. Ashkenani, "Effect of Date Extract on Growth and Spore Germination of Bacillus subtilis," Microbios, Vol. 59, No. 240-241, 1989, p. 203.

[15] W. Al-Shahib and R. Marshall, "The Fruit of the Date Palm: Its Possible Use as the Best Food for the Future?” International Journal of Food Sciences and Nutrition, Vol. 54, No. 4, 2003, pp. 247-259. doi:10.1080/09637480120091982

[16] H. Mirheydar, "Consumption of Plants in Prevention and Treatment of Disease,” Vol. 2, Tehran University, Persian, 1995, pp. 21-60.

[17] J. Malone, et al., "The Effect of Hyperglycemia on Nerve Conduction and Structure Is Age Dependent,” Diabetes, Vol. 45, No. 2, 1996, p. 209. doi:10.2337/diabetes.45.2.209

[18] D. Sigaudo-Roussel, B. Fromy and J. L. Saumet, "Diabetic Neuropathy in Animal Models,” Drug Discovery Today: Disease Models, Vol. 4, No. 1, 2007, pp. 39-44. doi:10.1016/i.ddmod.2007.09.005

[19] S. Usuki, et al., "Effect of Pre-Germinated Brown Rice Intake on Diabetic Neuropathy in Streptozotocin-Induced Diabetic Rats," Nutrition \& Metabolism, Vol. 4, No. 1, 2007, p. 25. doi:10.1186/1743-7075-4-25

[20] E. Liepinsh, et al., "Protective Effects of Mildronate in an Experimental Model of Type 2 Diabetes in Goto Kakizaki Rats,” British Journal of Pharmacology, Vol. 157, No. 8, 2009, pp. 1549-1556. doi:10.1111/j.1476-5381.2009.00319.x

[21] S. Sharma, S. Kulkarni and K. Chopra, "Effect of Resveratrol, a Polyphenolic Phytoalexin, on Thermal Hyperalgesia in a Mouse Model of Diabetic Neuropathic Pain,” Fundamental \& Clinical Pharmacology, Vol. 21, No. 1, 2007, pp. 89-94. doi:10.1111/j.1472-8206.2006.00455.X

[22] M. Shabani, et al., "Walnut Consumption Protects Rats against Cisplatin-Induced Neurotoxicity," NeuroToxicology, Vol. 33, No. 5, 2012, pp. 1314-1321. doi:10.1016/j.neuro.2012.08.004

[23] M. Shabani, et al., "Profound Destructive Effects of Adolescent Exposure to Vincristine Accompanied with Some Sex Differences in Motor and Memory Performance," Canadian Journal of Physiology and Pharmacology, Vol. 90, No. 4, 2012, pp. 379-386. doi:10.1139/y11-132

[24] M. Razavinasab, et al., "Pharmacological Blockade of TRPV1 Receptors Modulates the Effects of 6-OHDA on Motor and Cognitive Functions in a Rat Model of Parkinson's Disease,” Fundamental \& Clinical Pharmacology, 2012. doi:10.1111/fcp. 12015
[25] N. Zangiabadi, et al., "Date Fruit Extract Is a Neuroprotective Agent in Diabetic Peripheral Neuropathy in Streptozotocin-Induced Diabetic Rats: A Multimodal Analysis," Oxidative Medicine and Cellular Longevity, 2011, Article ID: 976948. doi:10.1155/2011/976948

[26] M. Shabani, et al., "Evaluation of Destructive Effects of Exposure to Cisplatin during Developmental Stage: No Profound Evidence for Sex Differences in Impaired Motor and Memory Performance,” The International Journal of Neuroscience, Vol. 122, No. 8, 2012, pp. 439-448.

[27] B. Galer, A. Gianas and M. Jensen, "Painful Diabetic Polyneuropathy: Epidemiology, Pain Description, and Quality of Life,” Diabetes Research and Clinical Practice, Vol. 47, No. 2, 2000, pp. 123-128. doi:10.1016/S0168-8227(99)00112-6

[28] M. Serpell, "Anatomy, Physiology and Pharmacology of Pain,” Anaesthesia \&Intensive Care Medicine, Vol. 6, No. 1, 2005, pp. 7-10. doi:10.1383/anes.6.1.7.57133

[29] E. Peschke, "Melatonin, Endocrine Pancreas and Diabetes,” Journal of Pineal Research, Vol. 44, No. 1, 2008, pp. 26-40.

[30] M. Tuzcu and G. Baydas, "Effect of Melatonin and Vitamin E on Diabetes-Induced Learning and Memory Impairment in Rats,” European Journal of Pharmacology, Vol. 537, No. 1-3, 2006, pp. 106-110. doi:10.1016/j.ejphar.2006.03.024

[31] D. Kooyenga, et al., "Palm Oil Antioxidant Effects in Patients with Hyperlipidaemia and Carotid Stenosis: 2 Year Experience,” Asia Pacific Journal of Clinical Nutrition, Vol. 6, No. 1, 1997, pp. 72-75.

[32] T. Ng, et al., "Nonhypercholesterolemic Effects of a Palm-Oil Diet in Malaysian Volunteers," American Journal of Clinical Nutrition, Vol. 53, No. 4, 1991, p. 1015 S.

[33] K. Sundram, T. Karupaiah and K. Hayes, "Stearic AcidRich Interesterified Fat and Trans-Rich Fat Raise the LDL/HDL Ratio and Plasma Glucose Relative to Palm Olein in Humans," Nutrition \& Metabolism, Vol. 4, No. 3, 2007, p. 3.

[34] M. Al-Farsi, et al., "Compositional and Sensory Characteristics of Three Native Sun-Dried Date (Phoenix dactylifera L.) Varieties Grown in Oman,” Journal of Agricultural and Food Chemistry, Vol. 53, No. 19, 2005, pp. 7586-7591.

[35] A. Allaith, “Antioxidant Activity of Bahraini Date Palm (Phoenix dactylifera L.) Fruit of Various Cultivars," International Journal of Food Science \& Technology, Vol. 43, No. 6, 2008, pp. 1033-1040. doi:10.1111/j.1365-2621.2007.01558.x

[36] J. Podratz, E. Rodriguez and A. Windebank, “Antioxidants Are Necessary for Myelination of Dorsal Root Ganglion Neurons, in Vitro,” Glia, Vol. 45, No. 1, 2004, pp. 54-58. doi:10.1002/glia.10302

[37] P. van Dam, et al., "Nerve Function and Oxidative Stress in Diabetic and Vitamin E-Deficient Rats,” Free Radical Biology and Medicine, Vol. 24, No. 1, 1998, pp. 18-26.

[38] M. Backonja, "Defining Neuropathic Pain,” Anesthesia \& Analgesia, Vol. 97, No. 3, 2003, p. 785. 
[39] S. Sayyed, A. Kumar and S. Sharma, "Effects of U83836 E on Nerve Functions, Hyperalgesia and Oxidative Stress in Experimental Diabetic Neuropathy,” Life Sciences, Vol. 79, No. 8, 2006, pp. 777-783. doi:10.1016/j.lfs.2006.02.033

[40] H. Kim, et al., "Analgesic Effect of Vitamin E Is Mediated by Reducing Central Sensitization in Neuropathic Pain,” Pain, Vol. 122, No. 2, 2006, pp. 53-62.

[41] G. Baydas, H. Canatan and A. Turkoglu, "Comparative Analysis of the Protective Effects of Melatonin and Vitamin E on Streptozocin-Induced Diabetes Mellitus,” Atherosclerosis, Vol. 2, 2002, pp. 8-10.

[42] M. Devi, Y. Suresh and U. Das, "Preservation of the Antioxidant Status in Chemically-Induced Diabetes Mellitus by Melatonin,” Journal of Pineal Research, Vol. 29, No.
2, 2000, pp. 108-115. doi:10.1034/j.1600-079X.2000.290207.X

[43] A. Maritim, R. Sanders and J. Watkins III, "Diabetes, Oxidative Stress, and Antioxidants: A Review,” Journal of Biochemical and Molecular Toxicology, Vol. 17, No. 1, 2003, pp. 24-38. doi:10.1002/jbt.10058

[44] M., Ebadi, et al., "Pineal Opioid Receptors and Analgesic Action of Melatonin,” Journal of Pineal Research, Vol. 24, No. 4, 1998, pp. 193-200.

[45] G. Maestroni, A. Conti and W. Pierpaoli, "Role of the Pineal Gland in Immunity: II. Melatonin Enhances the Antibody Response via an Opiatergic Mechanism,” Clinical and Experimental Immunology, Vol. 68, No. 2, 1987, p. 384. 